\title{
Sítio Metastático Incomum - Metástases Peritoneais em Melanoma Maligno: Relato de Caso
}

doi: https://doi.org/10.32635/2176-9745.RBC.2021v67n2.1239

\author{
Unusual Metastatic Site - Peritoneal Metastasis in Malignant Melanoma: Case Report \\ Sitio Metastásico Raro - Metástasis Peritoneales en Melanoma Maligno: Reporte de Caso
}

\author{
Amanda da Silva Anjos'; Diego Carvalho Duarte Mari²; Brenno Giovanni Hernando Vidottii ${ }^{3}$ Maikele Ertel ${ }^{4}$; John Carlos Claros Terraza5; \\ Bárbara Urnau Sfreddo ${ }^{6}$
}

\begin{abstract}
RESUMO
Introdução: $\mathrm{O}$ melanoma cutâneo é um tumor com grande capacidade de metastização. Um dos quadros possíveis, mas bastante raro, é quando a metástase se aloja em peritônio (carcinomatose peritoneal). Relato do caso: Paciente feminina, 61 anos, encaminhada por massa inguinal esquerda suspeita e lesão hipercrômica de $2 \mathrm{~cm}$, com área de regressão em membro inferior esquerdo. A paciente foi submetida à linfadenectomia inguinal esquerda e ressecçáo da lesão de pele, que evidenciou melanoma maligno cutâneo extensivo superficial invasor, de espessura de Breslow 1,2 mm, e nível de Clark II, bem como metástase linfonodal. O tratamento adjuvante incluiu radioterapia e quimioterapia. Durante follow-up, oito meses depois, ultrassonografia e tomografia computadorizada de abdome evidenciaram imagem nodular sólida hipoecoica, heterogênea e bosselada, mal definida e mal delimitada em projeção para-aórtica à esquerda, com 12,7x6,7x4,8 $\mathrm{cm}$. Em laparotomia exploradora, encontrou-se massa volumosa retroperitoneal em flanco esquerdo, com envolvimento do ângulo de Treitz e presença de carcinomatose peritoneal. O laudo anatomopatológico evidenciou neoplasia maligna pouco diferenciada epitelioide infiltrativa, compatível com história clínica de melanoma. A paciente evoluiu a óbito após consulta com oncologia clínica. Conclusáo: Existem poucos casos de metástase peritoneal de melanoma relatados na literatura. Contudo, deve-se levar em consideração sua possibilidade de ocorrência e as opçóes terapêuticas disponíveis, além de também compreender seu impacto no prognóstico do paciente. Apesar de rara, a carcinomatose peritoneal deve ser considerada quando pacientes com histórico pessoal de melanoma apresentarem queixas abdominais inespecíficas, como a paciente do caso relatado.
\end{abstract}

Palavras-chave: Melanoma; Peritônio; Metástase Neoplásica; Neoplasias Peritoneais.

\begin{abstract}
Introduction: Cutaneous melanoma is a tumor with great capacity for metastasis. One of the possible but quite rare scenarios is when the metastasis is lodged in the peritoneum (peritoneal carcinomatosis). Case report: 61-year-old female patient, referred by a suspected left inguinal mass and $2 \mathrm{~cm}$ hyperchromic lesion, with regression area in the left lower limb. The patient underwent left inguinal lymphadenectomy and resection of the skin lesion, which revealed invasive extensive superficial cutaneous malignant melanoma, Breslow depth $1.2 \mathrm{~mm}$ and Clark II level, as well as lymph node metastasis. Adjuvant treatment included radiotherapy and chemotherapy. During follow-up, 8 months later, abdominal ultrasound and computed tomography showed hypoechoic, heterogeneous, and beveled solid nodular image, poorly defined and delimited in left para-aortic projection, with $12.7 \times 6.7 \times 4.8 \mathrm{~cm}$. In exploratory laparotomy, a large retroperitoneal mass was found on the left flank, involving the Treitz angle, and revealed the presence of peritoneal carcinomatosis. The anatomopathological report showed a poorly differentiated malignant infiltrative epithelioid neoplasm, compatible with the clinical history of melanoma. The patient died after consultation with clinical oncology. Conclusion: There are few cases of peritoneal metastasis of melanoma reported in the literature. However, its possibility of occurrence and the therapeutic options available must be taken into account, in addition to understanding its impact on the patient's prognosis. Although rare, peritoneal carcinomatosis should be considered when patients with a personal history of melanoma have non-specific abdominal complaints, such as the patient in the case reported.

Key words: Melanoma; Peritoneum; Neoplasm Metastasis; Peritoneal Neoplasms.
\end{abstract}

\section{RESUMEN}

Introducción: El melanoma cutáneo es un tumor con gran capacidad de metástasis. Una de las situaciones posibles, pero bastante rara, es cuando la metástasis se aloja en el peritoneo. Relato del caso: paciente femenina de 61 años, remitida por sospecha de masa inguinal izquierda y lesión hipercrómica de $2 \mathrm{~cm}$, con área de regresión, en miembro inferior izquierdo. La paciente fue sometida a linfadenectomía inguinal izquierda y resección de la lesión cutánea, que demonstró melanoma maligno cutáneo superficial extenso invasivo, Breslow 1,2 mm y nivel de Clark II, así como metástasis ganglionares. El tratamiento adyuvante incluyó radioterapia y quimioterapia. Durante el seguimiento, 8 meses después, la ecografía y la tomografía computarizada de abdomen mostraron una imagen nodular sólida hipoecoica, heterogénea y biselada, mal definida y delimitada en proyección para-aórtica izquierda, con 12,7x6,7x4,8 cm. En la laparotomía exploradora se encontró una gran masa retroperitoneal en el flanco izquierdo, involucrando el ángulo de Treitz y presencia de carcinomatosis peritoneal. El informe anatomopatológico mostró una neoplasia epitelioide infiltrativa maligna mal diferenciada, compatible con la historia clínica de melanoma. El paciente falleció tras consulta con oncología clínica. Conclusión: Hay pocos casos de metástasis peritoneal de melanoma reportados en la literatura. Sin embargo, se debe tener en cuenta su posibilidad de ocurrencia y las opciones terapéuticas disponibles, además de comprender su impacto en el pronóstico del paciente. Aunque es poco común, la carcinomatosis peritoneal debe considerarse cuando los pacientes con antecedentes personales de melanoma tienen molestias abdominales inespecíficas, como la paciente del caso. Palabras clave: Melanoma; Peritoneo; Metastásis de la Neoplasia; Neoplasias Peritoneales.

${ }^{1}$ Faculdades Pequeno Príncipe. Curitiba (PR), Brasil. E-mail: anjosacademico@outlook.com. Orcid iD: https://orcid.org/0000-0002-8064-5161

${ }^{2-5}$ Hospital do Rocio. Campo Largo (PR), Brasil. E-mails: diegocdmari@gmail.com, bre_nno@hotmail.com; maykiertel@hotmail.com; john.c.claros.t@gmail.com; Orcid iD: https://orcid.org/0000-0002-0160-7686; Orcid iD: https://orcid.org/0000-0002-3239-6068; Orcid iD: https://orcid.org/0000-0001-5520-6055; Orcid iD; https://orcid.org/0000-0003-0150-2670

${ }^{6}$ Pontifícia Universidade Católica do Paraná. Curitiba (PR), Brasil. E-mail: barbarasfreddo@hotmail.com. Orcid iD: https://orcid.org/0000-0002-2901-1470 Endereço para correspondência: Amanda da Silva Anjos. Rua Izaias Belloni, 199 - Santa Felicidade. Curitiba (PR), Brasil. CEP 82020-600. E-mail: anjosacademico@outlook.com 


\section{INTRODUÇÃO}

O melanoma cutâneo maligno é um câncer agressivo capaz de metastizar rapidamente. A propagação sistêmica da doença pode ser direta, linfática ou hematogênica ${ }^{1}$. Implantes peritoneais provenientes de neoplasias primárias extra-abdominais são incomuns e correspondem a aproximadamente $10 \%$ dos casos de metástase em peritônio, como, por exemplo, câncer de mama ( $41 \%$ dos casos), câncer de pulmão (21\%) e melanoma maligno $(9 \%)^{2}$.

No estudo populacional de Flanagan et al. ${ }^{3}, 9,4 \%$ $(\mathrm{n}=543)$ dos pacientes com metástases peritoneais apresentavam uma neoplasia primária extra-abdominal, sendo que, destes, 9,3\% apresentavam como tumor primário o melanoma. Da população total, apenas $0,5 \%$ possuía melanoma com diagnóstico de implantes peritoneais; $75 \%$ dos pacientes eram do sexo masculino; e mais de $82 \%$ foram diagnosticados com metástases peritoneais após o diagnóstico do câncer primário. Neste estudo, o subtipo nodular foi o mais comum $(29 \%)^{3}$.

Poucos são os casos relatados na literatura. Lee et al. ${ }^{4}$ descreveram um caso de uma paciente que, após dez anos do diagnóstico e excisão de um melanoma, apresentou recorrência tumoral na forma de carcinomatose peritoneal ${ }^{4}$. McBride e Calhoun ${ }^{5}$ apresentaram um caso de melanoma anorretal que desenvolveu metástases peritoneais extensas.

No presente artigo, relata-se o caso de uma paciente com melanoma cutâneo que se apresentou inicialmente com linfonodomegalias inguinais e evoluiu posteriormente com metástase retroperitoneal e carcinomatose peritoneal.

\section{RELATO DO CASO}

Paciente feminina, 61 anos, encaminhada a um serviço de referência em oncologia por massa inguinal esquerda suspeita para neoplasia, em evolução por três meses. Foi realizada ultrassonografia (US) de partes moles da raiz da coxa esquerda na origem que evidenciou lesão nodular sólida heterogênea com áreas císticas associadas, medindo cerca de 5,6x4,5x4,4 cm. A massa inguinal, à palpação, revelou linfonodomegalia com cerca de $7 \mathrm{~cm}$ em porção anteromedial de coxa esquerda, e outra menor de $2 \mathrm{~cm}$.

A paciente apresentava lesão cutânea hipercrômica com área de regressão, de aproximadamente $2 \mathrm{~cm}$, compatível com melanoma, em porção posterolateral de membro inferior esquerdo, referindo mudança de padrão nos últimos meses.

Negou etilismo, tabagismo e histórico familiar de neoplasia. Histórico pessoal da paciente revelou hipertensão arterial sistêmica. Optou-se por ressecção da lesão de pele com anestesia local e rotação de retalho local, sem intercorrências.

O laudo anatomopatológico da peça evidenciou melanoma maligno cutâneo extensivo superficial invasor, de espessura de Breslow 1,2 mm e nível de Clark II. Não foram evidenciados infiltrados inflamatórios intra e peritumorais, nem ulceraçôes e satelitose, e as margens cirúrgicas estavam livres de neoplasia.

Tomografias computadorizadas (TC) de crânio, abdome e tórax sem particularidades. Não havia elevaçóes significativas dos marcadores tumorais carcinoembryonic antigen (CEA) e lactate dehydrogenase (LDH). O estadiamento da paciente no momento era de pT4N3MX (EC III). Decidiu-se, então, por realização de linfadenectomia inguinal esquerda, que evidenciou melanoma metastático extensamente necrótico infiltrativo em três linfonodos dos seis retirados. A paciente foi encaminhada para realização de quimioterapia com 12 ciclos de interferon e radioterapia de dose 48Gy em região inguinal esquerda.

Durante o follow-up, a paciente se queixou de dor epigástrica de forte intensidade. US de abdome total revelou imagem nodular hipoecoica heterogênea, medindo cerca de 3,9x2,2 cm em projeçáo retroperitoneal para-aórtica à esquerda. Foi solicitado TC de abdome total que náo demonstrou anormalidades e seguiu-se acompanhamento.

Em outra US de abdome total, realizada oito meses após a primeira, evidenciou-se imagem nodular sólida hipoecoica, heterogênea e bosselada, mal definida e mal delimitada, medindo cerca de $12,7 \times 6,7 \times 4,8 \mathrm{~cm}$ em projeção para-aórtica à esquerda, sem plano de clivagem bem definido com o bordo inferior da cauda pancreática e com parte das alças enterocólicas (Figura 1).

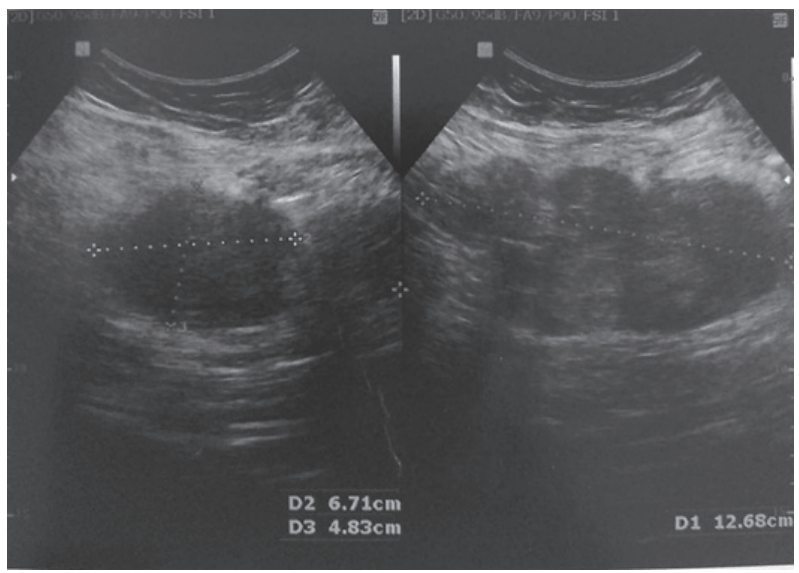

Figura 1. Ultrassonografia de abdome total evidenciando lesão em projeção para-aórtica à esquerda

A investigação foi complementada com TC de abdome total, com contraste, que evidenciou lesão heterogênea 
de aspecto expansivo, com realce heterogêneo, medindo $9,7 \times 6,7 \times 6,4 \mathrm{~cm}$, apresentando ampla comunicação com alças de delgado, não sendo possível excluir lesão primária (Figuras 2, 3 e 4). TC de crânio e tórax dentro da normalidade. O plano terapêutico foi laparotomia exploradora.
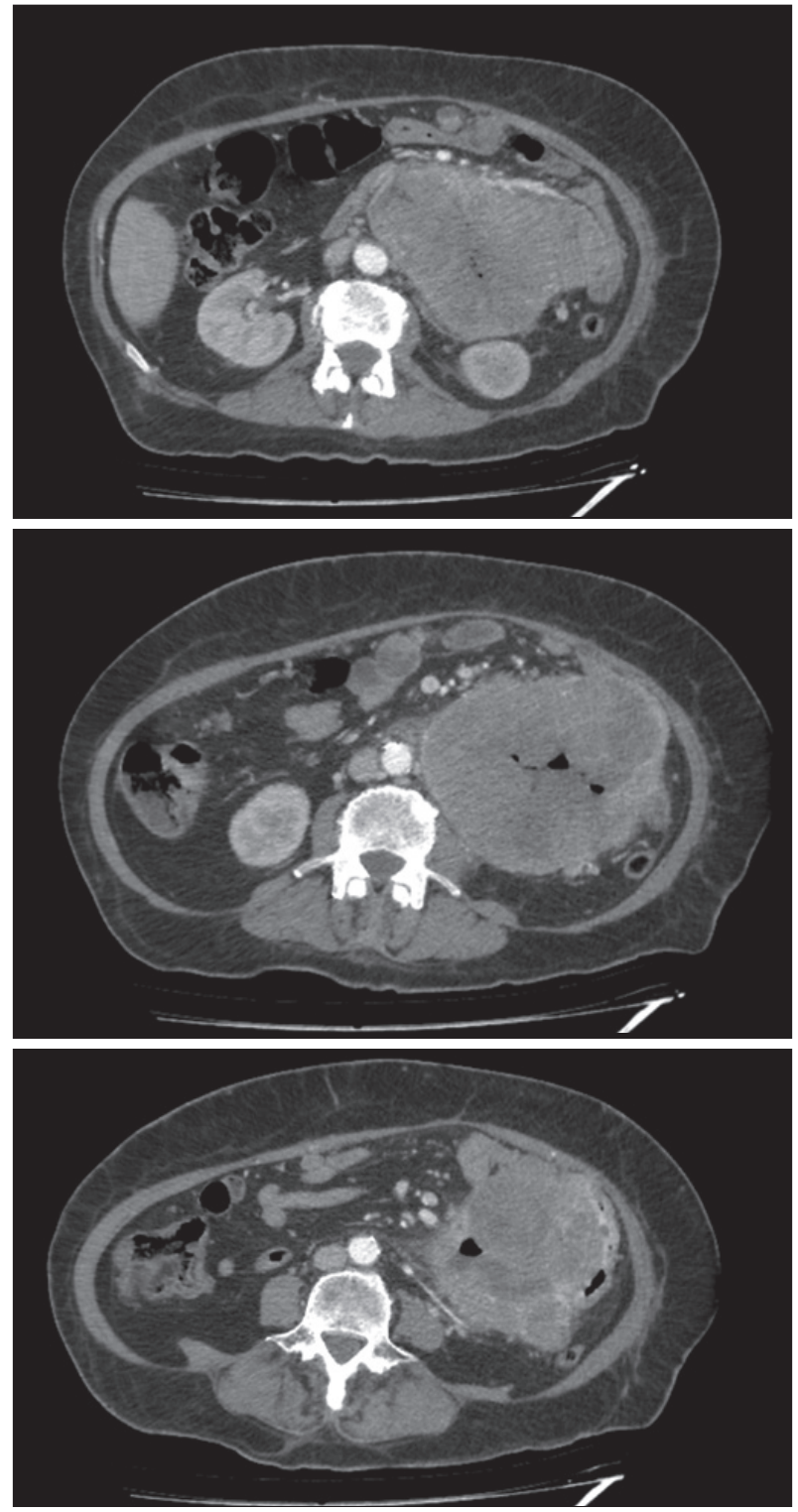

Figuras 2, 3 e 4 . Tomografia computadorizada de abdome com contraste, demonstrando lesão extensa em projeção para-aórtica esquerda

No intraoperatório, foi evidenciada massa volumosa retroperitoneal em flanco esquerdo, com envolvimento do ângulo de Treitz e presença de carcinomatose peritoneal (Figura 5). Foram realizadas biópsias de peritônio e epíplon e gastroenteroanastomose.

O laudo anatomopatológico das biópsias realizadas evidenciou neoplasia maligna pouco diferenciada epitelioide

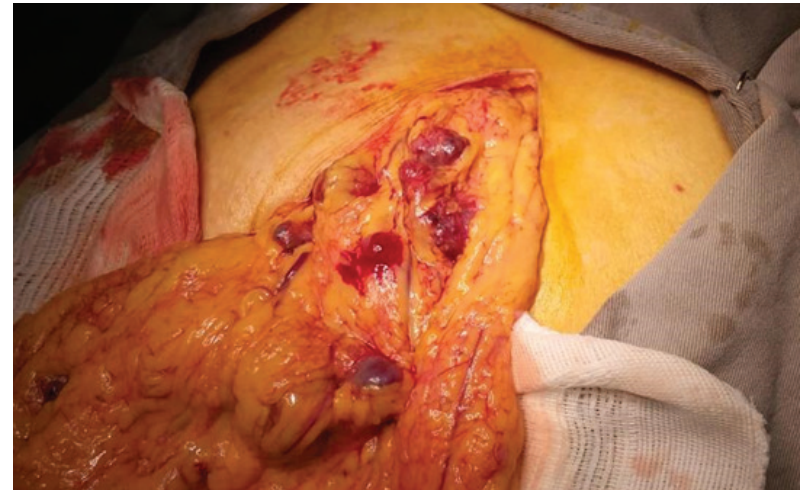

Figura 5. Implantes peritoneais evidenciados no intraoperatório

infiltrativa, compatível com história clínica de melanoma. Assim, a paciente recebeu um upstaging (T4N3M1, EC IV), sendo então encaminhada para a oncologia clínica. O plano terapêutico para a paciente foi novo esquema quimioterápico paliativo com 12 ciclos de DTIC (dacarbazina). A paciente não realizou o esquema proposto, pois foi a óbito 20 dias após a consulta com a oncologia clínica.

O estudo foi aprovado pela Comissão de Ética em Pesquisa do Hospital e Maternidade Angelina Caron, no Paraná, sob o número de parecer 4.304.361, de 28/9/2020 (CAAE 38271120.0.0000.5226).

\section{DISCUSSÃO}

O melanoma maligno é uma neoplasia agressiva com alto potencial metastático originada dos melanócitos, podendo ocorrer em diversos sítios, sendo mais frequente na pele ${ }^{6}$. Os sítios de metástase mais comuns são pulmão, fígado, linfonodos, cérebro e meninges, ossos e trato gastrointestinal ${ }^{6}$.

Quanto ao subtipo, o estudo de Flanagan et al. ${ }^{3}$ reportou que o melanoma nodular foi o subtipo que mais comumente desenvolveu implantes peritoneais ${ }^{3}$. Destaca-se que a paciente do caso relatado apresentava um melanoma do tipo extensivo superficial, que, segundo os autores ${ }^{3}$, seria um subtipo com menor propensão de desenvolver metástases peritoneais.

O termo carcinomatose peritoneal geralmente refere-se ao envolvimento metastático do peritônio, e comumente ocorre em casos de neoplasias gastrointestinais ou ginecológicas em estágio avançado com envolvimento locorregional ${ }^{2}$. $\mathrm{O}$ acometimento metastático peritoneal proveniente de tumores primários extra-abdominais é incomum ${ }^{4}$. Apesar disso, Lee et al. ${ }^{6}$ descreveram um caso de carcinomatose peritoneal derivada de um melanoma em uma paciente de 22 anos.

No caso da carcinomatose peritoneal por melanoma, câncer de pulmão e câncer de mama, a via de disseminação mais comum é a hematogênica² 
Em geral, pacientes com metástases peritoneais se encontram em um estágio avançado da doença ${ }^{2}$. Flanagan et al. $^{3}$ reportaram que a sobrevida global média dos pacientes com melanoma diagnosticados com implantes peritoneais foi de 1,8 meses (0-99,3), enquanto a sobrevida global média no grupo de pacientes com doença em estágio IV, porém sem acometimento peritoneal, foi de 12,3 meses $^{3}$.

Os dois achados clínicos mais importantes relacionados à presença de implantes peritoneais são ascite e obstrução intestinal, apesar de serem encontrados em menos de $50 \%$ dos pacientes. Outro sinal importante é a presença de células neoplásicas no líquido ascítico ${ }^{2}$. A paciente do caso se apresentou com dor epigástrica de forte intensidade, sendo uma variante dos achados clínicos que comumente estão relacionados com o acometimento peritoneal.

Esse envolvimento comumente é um achado incidental detectado durante exploração cirúrgica ou em exames de imagem como TC ou ressonância magnética (RM). A biópsia das lesôes suspeitas é capaz de confirmar o diagnóstico e diferenciar de possíveis condiçôes e tumores primários do peritônio, como linfomatose, tuberculose, pseudomixoma peritoneal, sarcomatose e mesotelioma ${ }^{2,5}$.

É importante realizar uma investigação apropriada no caso de metástases peritoneais, já que constituem uma das contraindicaçóes absolutas da cirurgia com intenção curativa. Além disso, é fundamental determinar a extensão, tamanho dos implantes e envolvimento de outros órgãos por metástases, a fim de avaliar o prognóstico do paciente ${ }^{2}$.

Por vezes, as lesões neoplásicas são visíveis na TC, RM e TC por emissão de pósitrons (PET-CT), utilizando 18-FDG (fluorodesoxiglicose). Os achados incluem espessamento focal ou difuso dos folhetos peritoneais, que podem ter aparência esclerótica, nodular, reticular, reticulonodular ou estruturas semelhantes a placas. Por vezes, uma densidade de grande dimensão e espessura fica visível entre a parede abdominal e as alças intestinais, estrutura chamada de omental cake ${ }^{2}$.

Com relaçáo à RM, esta não apresenta superioridade em relação à TC na detecção da carcinomatose peritoneal. O PET-CT identifica as lesôes neoplásicas baseado no uptake de glicose das células, tendo grande acurácia. A laparoscopia diagnóstica também se apresenta como uma ferramenta útil no diagnóstico da metástase peritoneal, já que pode evitar uma laparotomia desnecessária, apesar de muitos cirurgióes temerem a recorrência no sítio de inserção do trocater ${ }^{2}$.

Neste contexto, o não aparecimento dos implantes peritoneais na TC de abdome no follow-up da paciente é justificado pela limitação do exame quando esse tipo de neoplasia é localizado; ou seja, há pouca sensibilidade quando o sítio neoplásico é pequeno. Assim, o diagnóstico da carcinomatose peritoneal é complexo e o padrão-ouro é a visualização direta do peritônio, via laparotomia ou laparoscopia ${ }^{7}$.

A quimioterapia sistêmica é o padrão-ouro de terapia para pacientes com metástases a distância derivadas de melanoma, sendo que o quimioterápico mais utilizado é a dacarbazina, mesmo com uma taxa de resposta média de apenas $15 \%^{6,8}$. Quimioterapias combinadas podem aumentar a taxa de resposta quando comparadas com a monoterapia de dacarbazina, contudo, não aumentam a sobrevida dos pacientes e estáo associadas com maior toxicidade. Altas doses de interleucina- 2 ou interferon- $\alpha-$ $-2 b$ também podem ser usadas no tratamento do melanoma metastático ${ }^{6}$.

Em um cenário de doença avançada, pode-se, ainda, utilizar a imunoterapia no tratamento dos pacientes, como o nivolumab (anti-PD1), ipilimumab (antiCTLA4) e o pembrolizumab (anti-PD1), bem como terapias-alvo em caso de BRAF mutado, como dabrafenib, trametinib e vemurafenib ${ }^{9,10}$. No entanto, como possuem alto custo, seu uso é limitado. Em um contexto de carcinomatose peritoneal por melanoma maligno, os efeitos do nivolumab associado à citorredução cirúrgica e Hyperthermic Intraperitoneal Chemotherapy (HIPEC) foram estudados em modelo animal. No grupo que recebeu o nivolumab, o peritoneal carcinomatosis index (PCI) foi significativamente menor, bem como uma resposta linfocítica maior, menor tamanho tumoral e quantidade de nódulos, e menor escore de graduação da regressão peritoneal (maior taxa de resposta completa ao tratamento). Dessa forma, o tratamento com nivolumab em um cenário de melanoma maligno metastático tornase uma opçáo promissora ${ }^{8}$. O guideline da National Comprehensive Cancer Network (NCCN) ${ }^{10}$ atualizado em 2020 traz a monoterapia com agentes anti-PD1 e terapias-alvo para BRAF como terapia de primeira linha em casos de doença metastática ou irressecável ${ }^{10}$.

\section{CONCLUSÃO}

A carcinomatose peritoneal é um dos sítios incomuns de metástase do melanoma cutâneo maligno, tendo poucos casos relatados na literatura. Contudo, deve-se levar em consideração sua possibilidade de ocorrência e as opçóes terapêuticas disponíveis, além de também compreender seu impacto no prognóstico do paciente. Apesar de rara, a carcinomatose peritoneal deve ser considerada quando pacientes com histórico pessoal de melanoma maligno apresentarem queixas abdominais inespecíficas, como a paciente do caso relatado. 


\section{CONTRIBUIÇÕES}

Amanda da Silva Anjos e Diego Carvalho Duarte Mari contribuíram na concepção e/ou no planejamento do estudo; na obtençáo, análise e interpretação dos dados; assim como na redação e revisão crítica com contribuição intelectual. Bárbara Urnau Sfreddo contribuiu na redação e revisão crítica com contribuição intelectual. Brenno Giovanni Hernando Vidotti, Maikele Ertel e John Carlos Claros Terraza contribuíram na revisão crítica com contribuiçáo intelectual. Todos os autores aprovaram a versão final a ser publicada.

\section{DECLARAÇÃO DE CONFLITO DE INTERESSES}

Nada a declarar.

\section{FONTES DE FINANCIAMENTO}

Não há.

\section{REFERÊNCIAS}

1. De Vita F, Giordano G, Fabozzi A, et al. Metastatic melanoma: an unusual presentation. Tumori. 2011;97(1):130-2. doi: https://doi. org/10.1177/030089161109700124

2. StatPearls [Internet]. Treasure Island (FL): StatPearls Publishing; 2021 Jan. Desai JP, Moustarah F. Peritoneal Metastasis; 2020 [cited 2020 May 18]. Available from: https://pubmed.ncbi.nlm.nih.gov/31082158/ Cited in: PubMed; PMID: 31082158.

3. Flanagan M, Solon J, Chang KH, et al. Peritoneal metastases from extra-abdominal cancer - A populationbased study. Eur J Surg Oncol. 2018;44(11):1811-7. doi: https://doi.org/10.1016/j.ejso.2018.07.049

4. Lee EY, Heiken JP, Huettner PC. Late recurrence of malignant melanoma presenting as peritoneal "carcinomatosis". Abdom Imaging. 2003;28(2):284-6. https://doi.org/10.1007/s00261-002-0039-x

5. McBride M, Calhoun S. Peritoneal Carcinomatosis Arising from Primary Anorectal Melanoma. J Radiol Case Rep. 2019;13(4):28-37. doi: https://doi.org/10.3941/ jrcr.v13i4.3458

6. Lee ES, Ahn JH, Lee TS, et al. Metastatic malignant melanoma with peritoneal seeding in a young woman: a case report. Obstet Gynecol Sci. 2014;57(3):240-3. doi: doi: https://doi.org/10.5468/ogs.2014.57.3.240

7. Coccolini F, Gheza F, Lotti M, et al. Peritoneal carcinomatosis. World J Gastroenterol. 2013;19(41):697994. doi: https://doi.org/10.3748/wjg.v19.i41.6979

8. Duzgun O, Sarici IS, Gokcay S, et al. Effects of nivolumab in peritoneal carcinamatosis of malign melanoma in mouse model. Acta Cir. Bras. 2017;32(12):1006-12. doi: https://doi.org/10.1590/s0102-865020170120000002

9. Munhoz R, Wainstein A, Melo A, et al. Diretrizes de tratamentos oncológicos recomendados pela Sociedade Brasileira de Oncologia Clínica: melanoma cutâneo [Internet]. São Paulo: SBOC; 2020 [acesso 2020 maio 18]. Disponível em: https://www.sboc.org.br/images/ diretrizes/diretrizes_pdfs/2020/lote5/Diretrizes-SBOC2020---Melanoma-PS-PUBLICAO.pdf

10. National Comprehensive Cancer Network. Clinical practice guidelines in oncology: cutaneous melanoma. Plymouth Meeting, PA: NCCN; 2020. 